\title{
Thalassemia and COVID-19: Susceptibility and Severity
}

\author{
Somayeh Rahimi ${ }^{1}$, Saba Zakeri ${ }^{1}$, Mahsa Nouri ${ }^{1}$, Yaser Mohassel ${ }^{1}$, Bahareh Karami $^{1}$, Seyedeh Ozra \\ Hosseini Jomor ${ }^{1}$, Babak Sayad (iD) ${ }^{2}$, Zeinab Mohseni Afshar ${ }^{2}$, Zohreh Rahimi (iD ${ }^{3,{ }^{*}}$ and Zahra Asadi ${ }^{1}$ \\ ${ }^{1}$ Students Research Committee, Kermanshah University of Medical Sciences, Kermanshah, Iran \\ ${ }^{2}$ Infectious Diseases Research Center, Kermanshah University of Medical Sciences, Kermanshah, Iran \\ ${ }^{3}$ Department of Clinical Biochemistry, Kermanshah University of Medical Sciences, Kermanshah, Iran \\ ‘Corresponding author: Behavioral Research Center, Kermanshah University of Medical Sciences, Kermanshah, Iran. Email: rahimizus@yahoo.com
}

Received 2021 September 28; Revised 2021 November 06; Accepted 2021 November 13.

\begin{abstract}
Context: COVID-19 results in an imbalance between procoagulant and anticoagulant homeostatic mechanisms that could be complicated with thrombotic events. In $\beta$-thalassemia patients, the presence of comorbidities, iron overload, adrenal hypofunction, splenectomy, and chronic hypercoagulable state might increase the susceptibility to COVID-19 and its severity.

Evidence Acquisition: The search was conducted in PubMed, Web of Science, and Scopus databases for the key terms of $\beta$ thalassemia/thalassemia and COVID-19 until July 2021.

Results: The survey of published observational studies (mostly multicenter and case reports) indicated a lower prevalence of COVID19 in $\beta$-thalassemia patients compared with the general population, as well as mild to moderate COVID-19 in these patients, especially in those without comorbidity. $\beta$-Thalassemia children were susceptible to COVID-19 but with less severity compared to adults. There is no report of pulmonary embolism and thrombotic events in $\beta$-thalassemia patients with COVID-19; however, coagulation abnormality and pulmonary microembolism have been found in these patients.

Conclusions: Findings could be interpreted by the presence of high hemoglobin $\mathrm{F}(\mathrm{HbF}$ ) levels, the advantage of hydroxyurea (HU) therapy, splenectomy, and iron chelation therapy in these patients. However, due to the low sample size and studying mainly young patients, the results should be interpreted with caution, and it still needs more studies with a larger sample size to confirm these findings.
\end{abstract}

Keywords: Beta-thalassemia, SARS-CoV-2, COVID-19, Comorbidity, Iron Overload, Splenectomy, Hemoglobinopathies

\section{Context}

SARS-CoV-2 (severe acute respiratory syndrome coronavirus 2), which is the cause of COVID-19 (coronavirus disease 2019), infects the cells through binding to angiotensin-converting enzyme 2 . In response to the infection, $\mathrm{T}$ cell activation and cytokine storm occur in a severe form of the disease, resulting in organ damage, mainly lung damage (1). The pandemic of COVID-19 as a global public health emergency causes concern about patients with hemoglobinopathies, including thalassemia. Patients with $\beta$-thalassemia major need regular blood transfusions; although the risk of SARS-CoV-2 transmission through blood transfusion has not been documented, the risk of transmission in a viremic donor with high viral load should be considered.

Further, many $\beta$-thalassemia major patients are splenectomized. The role of splenectomy in the increased risk of viral infection or its severity is not clear, and specific data related to SARS-CoV-2 are absent (2). Although $\beta$-thalassemias are not generally associated with respiratory abnormalities, similar to SARS-CoV-2 patients in hemoglobin $(\mathrm{Hb})$ disorders, organ complications (such as heart and lung complications) and also immune system involvement have been detected (3). The presence of comorbidities (such as hypertension, heart disease, diabetes mellitus, and abnormal liver) and poor kidney function increase the severity of COVID-19 $(3,4)$. Therefore, severe organ dysfunctions might complicate SARS-CoV-2 infection in patients with hemoglobinopathies (3).

The present review aimed to summarize the prevalence and severity of COVID-19 in $\beta$-thalassemia patients infected with SARS-CoV-2 reported in the literature, as well as discuss the underlying factors involved in possible differences in the prevalence and severity of COVID-19 in these patients. Table 1 summarizes what is known about COVID19 susceptibility and severity in $\beta$-thalassemia patients and demonstrates how the current review will contribute to the field and future research on the subject. 


\begin{tabular}{lll}
$\begin{array}{l}\text { Table 1. Knowledge Related to the Susceptibility and Severity of COVID-19 in } \beta \text {-Thalassemia Patients and How the Current Review Will Contribute to the Field and Future } \\
\text { Research on the Subject }\end{array}$ & What Is New? & $\begin{array}{l}\text { What Are the Future Key Questions for Future } \\
\text { Work on the Topic? }\end{array}$ \\
\hline $\begin{array}{l}\text { What Is Known About the Topic? } \\
\begin{array}{l}\beta \text {-Thalassemia patients have comorbidities and } \\
\text { adrenal hypofunction that might make them } \\
\text { vulnerable to viral infection. }\end{array}\end{array}$ & $\begin{array}{l}\text { There is a lower prevalence of COVID-19 in } \\
\beta \text {-thalassemia patients compared with the general } \\
\text { population, as well as mild to moderate COVID-19 in } \\
\text { these patients. }\end{array}$ & $\begin{array}{l}\text { More studies with larger sample sizes need to } \\
\text { confirm lower susceptibility and the severity of } \\
\text { COVID-19 in } \beta \text {-thalassemia patients. }\end{array}$ \\
$\begin{array}{l}\beta \text {-Thalassemia patients have a chronic } \\
\text { hypercoagulable state. Also, COVID-19 could be } \\
\text { complicated with thrombotic events. }\end{array}$ & $\begin{array}{l}\text { High HbF levels, HU therapy, splenectomy, and iron } \\
\text { chelation therapy in } \beta \text {-thalassemia patients might } \\
\text { be beneficial against COVID-19 and its severe forms. }\end{array}$ & $\begin{array}{l}\text { The exact role and mechanism of high HbF levels, HU } \\
\text { therapy, splenectomy, and iron chelation therapy in } \\
\text { reduced prevalence and the severity of COVID-19 } \\
\text { need to be elucidated. }\end{array}$ \\
\hline
\end{tabular}

\section{1. $\beta$-Thalassemia and COVID-19}

$\beta$-Thalassemia is an inherited autosomal recessive disorder resulting from the decreased or the absence of $\beta$ globin chain synthesis. According to the clinical manifestations, $\beta$-thalassemia is categorized into $\beta$-thalassemia minor, $\beta$-thalassemia intermedia, and $\beta$-thalassemia major (5). In $\beta$-thalassemia major, regular blood transfusions can decrease anemia-related complications, compensate for bone marrow expansion, and improve the survival rate of patients. Iron chelation therapy reduces iron accumulation secondary to multiple transfusions and dramatically improves the prognosis (6).

There is an increased risk of thrombosis in patients with $\beta$-thalassemia intermedia and $\beta$-thalassemia major. The existence of a chronic hypercoagulable state, platelet activation, and enhanced thrombin generation (already existed in early childhood) has been observed in these patients (7).

The presence of comorbidities and severe iron overload might predispose $\beta$-thalassemia patients to virus infection. Excess blood iron load results from the nontransferrin-bound iron entrance into some cells and conversion to ferritin and hemosiderin over time, all of which occur in the setting of frequent transfusion. The influence of excessive iron on the hypothalamus or adrenal glands results in adrenal hypofunction, limiting the ability to fight infections (8). COVID-19 leads to systemic inflammatory response and imbalance between procoagulant and anticoagulant homeostatic mechanisms and is therefore complicated by thrombotic events (4).

Regarding the susceptibility of $\beta$-thalassemia patients to COVID-19 and its increased severity, there are 2 hypotheses. In hypothesis $1, \beta$-thalassemia patients might be more susceptible to COVID-19 and its severe form due to their chronic comorbidities, such as iron overload, stress erythropoiesis, diabetes, heart disease, liver disease, adrenal insufficiency, and splenectomy. Splenectomy and oxidative stress due to iron overload can cause an immunocompromised condition in $\beta$-thalassemia patients. Moreover, impaired maturation and decreased neutrophil ac- tivity, decreased natural killer cells, decreased phagocytic function of monocytes and macrophages, decreased $\mathrm{CD} 4^{+}$ to $\mathrm{CD}^{+}$ratio, and an increase in inflammatory cytokines have been observed in these patients (9).

In addition, these patients need continuous treatment and consequently increased contact with healthcare systems and hospitals, which put them in high exposure to SARS-CoV-2. Host cell oxidative stress plays a vital role in COVID-19 infection. Also, uncontrolled oxidative stress induces hemolytic anemia in patients with hemoglobinopathies. Further, therapy can induce hemolytic crises in patients with hemoglobinopathies, which should be avoided or closely monitored (10).

In hypothesis 2 , the heterozygous $\beta$-thalassemia patients might have immunity against SARS-CoV-2 infection. Using multiple linear regression analysis and relying only on statistical methods, the evolution of COVID-19 infection in 3 Italian regions of Puglia, Sardinia, and Sicilia with different prevalences of $\beta$-thalassemia was analyzed. It was proposed that heterozygous $\beta$-thalassemia individuals might develop immunity to SARS-CoV-2 infection (11).

\subsection{Red Blood Cells and COVID-19}

Red blood cells (RBCs) transport oxygen and could play a role in the severity of hypoxemia in patients with COVID19. In COVID-19 patients, increased levels of glycolytic intermediates and altered lipid metabolism without alterations in RBC count, hematocrit, or mean corpuscular $\mathrm{Hb}$ levels have been reported (12). However, in patients with severe COVID-19, the Hb levels essentially decreased compared with those with mild COVID-19; also, the association of poor outcomes of COVID-19 with higher $\mathrm{Hb}$ levels has been detected (13).

Among sickle cell disease (SCD) patients (including sickle $\beta$-thalassemia with COVID-19), the benefit of early simple/exchange transfusion in those patients with acute chest syndrome has been indicated with rapid clinical improvement of tachypnea/dyspnea and oxygen saturation and the clinical course of the disease (14). Also, the mortality rate of non-transfusion-dependent thalassemia 
(NTDT) patients was higher than transfusion-dependent thalassemia (TDT) patients (15). Further, the level of HbF might affect the outcome of COVID-19 in $\beta$-thalassemia patients. Unique structural properties of the HbF tetramer make HbF-containing RBCs inadequate hosts for Plasmodium falciparum (16). Thus, it is possible that high HbF levels in $\beta$-thalassemia patients provided an advantage against viral infection, particularly infection with SARS-CoV-2.

\section{Evidence Acquisition}

We searched PubMed, Web of Science, and Scopus databases for the key terms of $\beta$-thalassemia/thalassemia and COVID-19 until July 2021. A total of 13 published papers that consisted of 123 adult and children patients reported in questionnaire-based studies, observational studies, and case reports were summarized. The following data (if available) were extracted: sample size, mean age, history of splenectomy, type of $\beta$-thalassemia, splenectomy, transfusion dependency, presence of comorbidity, serum ferritin, and clinical course of COVID-19.

\section{Results}

\subsection{Observed/Reported Incidence and the Severity of COVID-19}

\subsubsection{Multicenter Studies}

Although patients with $\beta$-thalassemia are susceptible to infectious diseases due to immune system defects, splenectomy, adrenal hypofunction, iron overload, and multiple transfusions, a resistance to COVID-19 has been detected in $\beta$-thalassemia patients with a low prevalence of infection in Ferrara, a town in northern Italy with a high prevalence of $\beta$-thalassemia and COVID-19 affected individuals (17). Also, a preliminary report from northern Italy with a high prevalence of COVID-19 demonstrated that only 11 out of $6900 \beta$-thalassemia patients were detected with COVID-19 (18). Among infected patients with COVID-19, 10 patients were transfusion-dependent, and almost all patients had comorbidities. Despite the presence of comorbidities (especially splenectomy in these patients), severe manifestations of the disease, cytokine storm, and mortality were not observed in these patients, and the infected individuals had mild to moderate clinical presentations of COVID-19 (18).

Furthermore, in a multicenter study from Iran (including 18350 thalassemia major and intermedia that among them 15950 were transfusion-dependent), it was reported only 23 cases with COVID-19 (15 confirmed and 8 suspected symptomatic cases) (19). The severity of the disease was mild to moderate in 17 out of 23 patients, and 6 (26.1\%) patients died. However, 4 out of 15 confirmed COVID-19 died
(26.6\%), all of whom had comorbidities. In this study, most of the patients (60\%) had at least 1 comorbidity. Although $80 \%$ of confirmed COVID-19 patients were splenectomized, splenectomy was not significantly correlated with the fatal outcome of COVID-19. The results of this study suggested that the incidence of COVID-19 in patients with $\beta$ thalassemia major and intermedia was lower than that of the general population (19). However, the high mortality rate in these patients was attributed to the presence of comorbidities.

Furthermore, in an international multicenter study 9499 patients with hemoglobinopathies from 10 countries (including 2659 thalassemia major and 530 thalassemia intermedia) were surveyed using a questionnaire. In this study, there were 13 young patients ( 7 with thalassemia major, 3 with thalassemia intermedia, and 3 with SCD) with confirmed COVID-19. In the majority of patients, the clinical course of COVID-19 was moderate. However, there was 1 female $\beta$-thalassemia patient who died (20). The severity of COVID-19 was mild to moderate among thalassemia patients, but the presence of multiple comorbidities led to severe forms of the infection and poor prognosis.

In a study of 43 patients with $\beta$-thalassemia (nontransfusion- and transfusion-dependent) with at least 1 comorbidity from Iran (with the age range of 9-67 years), the mortality rate of NTDT patients (27.3\%) was higher than TDT patients (15.6\%) and the general population (4.71\%). In NTDT patients, there are potentially persistent chronic anemia and hypercoagulable state, and microthrombosis makes NTDT patients susceptible to developing pulmonary artery hypertension and heart failure, followed by a higher risk of death. Nonetheless, the prevalence of COVID-19 seems to be lower in $\beta$-thalassemia patients than in the general population (15).

In another report from this group (a nationwide Iranian experience), among $48 \beta$-thalassemia patients (34 were transfusion-dependent) with the age range of $9-67$ years, there were 8 severe/critical cases who had comorbidities and died (16.7\%) from COVID-19. They suggested a significantly lower prevalence and a higher mortality rate of COVID-19 in $\beta$-thalassemia patients compared with the general Iranian population. Also, they found susceptibility of children to COVID-19, but with less severity compared with adults. Further, they did not detect pulmonary embolism and thrombotic events in these patients during hospitalization (21).

People with chronic illness take care of themselves more than the healthy population. The less common prevalence of COVID-19 in thalassemia patients may be due to this reason. In a national study from England, among $26 \beta$-thalassemia patients with COVID-19,1TDT patient who had comorbidity (splenectomy) died (22). A questionnaire- 
based study evaluated the impact of COVID-19 on the medical and social care of $\beta$-thalassemia patients in Iran. According to this study, 11 out of $15 \beta$-thalassemia patients with COVID-19 recovered from COVID-19, and 4 died ( 2 with $\beta$-thalassemia major and 2 with $\beta$-thalassemia intermedia). Two out of $4 \beta$-thalassemia patients who died had comorbidities (diabetes and liver failure) (23). The data of studies that reported $\beta$-thalassemia patients infected with SARS-CoV-2 are presented in Table 2.

\subsubsection{Case Reports}

Mild COVID-19 was reported among 4 transfusiondependent $\beta$-thalassemia children from Indonesia. However, coagulation abnormality was detected in 1 patient without developing clinical thrombosis and with elevated D-dimer (24). In 2 young Pakistani patients, mild SARS-CoV-2 infection was reported $(10,25)$. One patient was a $\beta$-thalassemia major transfusion-dependent woman with a history of splenectomy without receiving specific COVID-19 treatment and supportive therapy (25), and the other patient was an asymptomatic male with $\mathrm{HbD} /$ thalassemia/G6PD deficiency without comorbidity (10). Also, mild COVID-19 infection in a 59-year-old transfusion-dependent female with $\beta$-thalassemia major was reported (26).

Further, SARS-CoV-2 infection was reported in an adult TDT patient who had undergone splenectomy and cholecystectomy many years ago and was treated with iron chelators. Despite developing pulmonary microembolism and requiring more transfusions, this patient recovered completely and was released from the hospital (27). Finally, Pinto et al. reported a splenectomized man with $\beta$ thalassemia major and severe pulmonary arterial hypertension who recovered after 10 days of hospitalization for COVID-19 (Table 2) (28). As indicated in Table 2, the severity and mortality due to COVID-19 were less in $\beta$-thalassemia children than in adults.

\subsection{Factors Affecting the Susceptibility and Severity of COVID-19}

\subsubsection{Hemoglobin Structure and Hemoglobin F Level}

In silico analysis of ORF10, ORF3a, and ORF1ab proteins of SARS-CoV-2 has indicated that these proteins can attack the beta chain of $\mathrm{Hb}$, resulting in the separation of iron from the porphyrin ring (29) with the consequence of impaired oxygen transfer. Thus, in hemoglobinopathies with beta chain defects (such as $\beta$-thalassemias), the virus might not attach to RBCs, disrupting the infection process (29). Also, the distant amino acid sequence of the SARSCoV-2 spike glycoprotein cytoplasmic tail is similar to the hepcidin protein (30). This glycoprotein can mimic the hepcidin function, enhance the entry of iron into the cell, and elevate ferritinemia. Thus, in hemoglobinopathies (such as thalassemia), due to defects or the absence of beta chains and low hepcidin level, susceptibility to COVID-19 infection might decrease (31).

Inconsistent with these findings, few studies have ruled out a link between the structure of $\mathrm{Hb}$ and the severity of COVID-19 disease. The Hb virus attachment theory has not been generally accepted (32), and experimental data are needed to confirm it. Further, Park et al (33) showed that hypoxemia in COVID-19 patients was unlikely to be due to impaired $\mathrm{O}_{2}$ handling in RBCs. E. Sotoudeh and H. Sotoudeh (34) found that mortality rates were lower in parts of the world where hemoglobinopathies were prevalent than in other parts. Therefore, it is suggested that the structure of $\mathrm{Hb}$ might be involved in the pathophysiology of COVID-19.

High levels of $\mathrm{HbF}$ in thalassemia patients, along with its antiparasitic effect, might be protective against viral infections such as SARS-CoV-2. The high prevalence of hemoglobinopathies in tropical countries with less COVID19-related mortality has been attributed to high HbF levels and hydroxycarbamide (hydroxyurea, [HU]) therapy, which induces $\mathrm{HbF}$ (34). Moreover, high levels of $\mathrm{HbF}$ and its protective role in patients with hemoglobinopathies and children could be the reason for less severe SARS-CoV2 infection. Thus, it was hypothesized that HbF-inducing drugs (such as HU and erythropoietin) could play a role in the control and treatment of COVID-19 (34).

$\mathrm{HU}$ is a medication used in patients with thalassemia intermedia, which has possible immune-compromising effects that might contribute to the adverse outcome in these patients. Short-term toxicities (neutropenia and thrombocytopenia) of HU have been demonstrated to be reversible and manageable. On the other hand, its antiinflammatory function, antiviral effect, and increased $\mathrm{HbF}$ levels might suggest the benefit of $\mathrm{HU}$ against the severe forms of COVID-19 $(35,36)$. The overall favorable outcome of COVID-19 in SCD children (including sickle/ $\beta$ thalassemia) has been attributed to the possible benefits of HU therapy in these patients (14). However, further evidence and clinical trials are needed to confirm the protective role of HU against the severity of COVID-19.

\subsubsection{Splenectomy and Iron Overload}

Splenectomy is a common therapeutic intervention in $\beta$-thalassemias. Since splenectomy reduces the removal of procoagulant RBCs and active platelets, it might increase the risk of coagulopathy (27). Despite the immunological functions of the spleen, there is no evidence of a higher risk of severe COVID-19 in asplenic/hyposplenic patients (20). A multicenter study from Iran (19) reported a high mortality rate of $\beta$-thalassemia patients with COVID-19. In this study, 
Rahimi Set al.

\begin{tabular}{|c|c|c|c|c|c|c|}
\hline Reference & Sample Size, N & Mean Age (Range), $y$ & $\begin{array}{l}\text { COVID-19 Severity } \\
\text { (Mortality), } N\end{array}$ & $\begin{array}{l}\text { Disease Type } \\
\text { (TDT:NTDT), N }\end{array}$ & $\begin{array}{l}\text { The Presence of } \\
\text { Comorbidity }^{\mathrm{a}}, \mathrm{N}\end{array}$ & $\begin{array}{l}\text { Serum Ferritin Mean } \\
\text { (Range), ng/mL }\end{array}$ \\
\hline (10) & 1 & 26 & 0 & $0: 1$ & 0 & 450 \\
\hline (18) & 11 & $44,31-61$ & 0 & $10: 1$ & 11 & NA \\
\hline (19) & 15 & $36.1,22-66$ & 4 & $12: 3$ & 12 & 1725 \\
\hline (20) & 10 & $35.7,22-66$ & 1 & 10:0 & 8 & $1653,225-5960$ \\
\hline (21) & 48 & $35.1,9-67$ & 8 & $34: 14$ & 38 & $1148,142-17000$ \\
\hline (22) & 26 & $33,0.5-92$ & 1 with comorbidity & 20:6 & NA & NA \\
\hline (23) & 4 & 30.5 & 4 & $4: 0$ & 4 & 1570 \\
\hline (24) & 4 & 9-17 & 0 & 4:0 & 0 & 2524 \\
\hline (25) & 1 & 25 & 0 & 1:0 & 1 & 3214 \\
\hline (26) & 1 & 59 & 0 & 1:0 & NA & NA \\
\hline (27) & 1 & 46 & 0 & 1:0 & 1 & $465-1337$ \\
\hline (28) & 1 & 57 & 0 & 1:0 & 1 & $\mathrm{NA}$ \\
\hline
\end{tabular}

Abbreviations: TDT, transfusion-dependent thalassemia; NTDT, non-transfusion-dependent thalassemia.

${ }^{a}$ Cancer, diabetes, liver failure, splenectomy, heart disease, pulmonary artery hypertension, hypertension, chronic kidney disease, nephropathy, and endocrine complications

approximately $82 \%$ of patients were splenectomized; however, the fatal outcome of COVID-19 was not associated with splenectomy (19). The anti-inflammatory effect and inflammatory cytokine reduction have been reported in an animal study after splenectomy. However, the beneficial role of splenectomy against cytokine storms and severe forms of COVID-19 in patients with $\beta$-thalassemia needs to be confirmed (37).

High ferritin levels could be a negative prognostic factor in patients with COVID-19, and iron chelation with deferiprone or deferoxamine (which has also been suggested in the treatment of COVID-19) decreased viral replication and related pro-inflammatory pathways (31). Therefore, iron chelation in patients who need transfusion might also be beneficial against COVID-19, which needs to be confirmed.

\subsection{Management of Patients with $\beta$-thalassemias}

Both intravascular and extravascular hemolysis can occur in thalassemia patients. Therefore, the cell blood count of thalassemia patients with COVID-19 needs to be closely monitored, and caution should be maintained toward the possibility of exacerbated hemolytic anemia in acute viral infection (20).

Although SARS-CoV-2 usually affects the respiratory tract, it may sometimes enter the circulation. Therefore, the possible risk of SARS-CoV-2 transmission through blood transfusion products should be considered. Although there is still no evidence of virus transmission through the blood transfusion and the absence of SARSCoV-2 viremia in a blood donor with COVID-19 4 days before the onset of symptoms has been reported, individuals with chronic transfusions should be aware of this possible risk and need to take precautions $(38,39)$. Theoretically, an asymptomatic infected blood donor may spread the virus unintentionally; therefore, it is recommended that COVID19 patients avoid blood donations for at least 28 days after being asymptomatic and completing medication (8).

Many patients with hemoglobinopathies, including those with thalassemias, could be at increased risk of developing severe complications of COVID-19. Therefore, the management of patients with hemoglobinopathies is important in controlling their infection. In the management of hemoglobinopathies (especially patients with thalassemias), lifestyle and nutrition controls should also be considered. Sufficient amounts of antioxidant vitamins $\mathrm{E}$ and $\mathrm{C}$ are necessary for $\beta$-thalassemia patients due to their need to be protected against oxidative stress, suppress the cytokine storm in acute respiratory distress syndrome, and improve antiviral potency. On the other hand, vitamin D3 as a regulator of adaptive and innate immunity has antiviral effects, and vitamin D deficiency could increase the mortality rate of COVID-19. Since iron accumulation in the liver and skin of $\beta$-thalassemia patients could disturb vitamin $\mathrm{D}$ synthesis, these patients might have vitamin $\mathrm{D}$ deficiency. Therefore, vitamin $\mathrm{D}$ supplementation in $\beta$ thalassemia patients is beneficial against viral and bacterial infections (40).

Two trace elements of zinc and selenium are involved 
in the immune system's integrity. Also, selenium is known to have anti-inflammatory and antiviral functions through altering the cytokine profile of macrophages and by $\mathrm{T}$ helper proliferation. Iron chelating agents can increase the excretion of these minerals and lead to deficiency. Hence, dietary supplementation of these elements is also necessary for $\beta$-thalassemia patients, particularly during the COVID-19 pandemic (40).

\section{Conclusions}

In $\beta$-thalassemia patients, the presence of comorbidities might make them vulnerable to viral infection. In addition, iron overload in these patients affects the hypothalamus or adrenal glands with consequent adrenal hypofunction, limiting the ability to fight infections. Further, there is a chronic hypercoagulable state, platelet activation, and enhanced thrombin generation in these patients. On the other hand, COVID-19 results in systemic inflammatory response and imbalance between procoagulant and anticoagulant homeostatic mechanisms, and it could be complicated with thrombotic events.

The survey of published observational studies (mostly multicenter and case reports) indicated a lower prevalence of COVID-19 in $\beta$-thalassemia patients compared with the general population, as well as mild to moderate COVID19 in these patients, especially in those patients without comorbidity. Above findings could be interpreted by the presence of high $\mathrm{Hb}$ F level (unsuitable $\mathrm{Hb}$ for infectious agents), the beneficial effect of HU therapy in reducing inflammatory and coagulation parameters, the possible role of splenectomy by decreasing proinflammatory cytokines, and decreased iron sources for viral replication due to iron chelation therapy in these patients. However, more studies with larger sample sizes are still needed to confirm these findings.

\section{Footnotes}

Authors' Contribution: Study concept and design: Z. Rahimi. Drafting of the manuscript: S. Rahimi, Zakeri, Nouri, Mohassel, Karami, Hosseini Jomor, Asadi, Z. Rahimi. Critical revision of the manuscript for important intellectual content: Sayad, Mohseni Afshar.

Conflict of Interests: The authors declare that they have no conflicts of interest.

Funding/Support: The present study did not receive any funding support.

\section{References}

1. Ebrahimi A, Sayad B, Rahimi Z. COVID-19 and psoriasis: biologic treatment and challenges. J Dermatolog Treat. 2020:1-5. doi: 10.1080/09546634.2020.1789051. [PubMed: 32598204].
2. Canatan D, De Sanctis V. The medical concerns of patients with thalassemias at the time of COVID-19 outbreak: The personal experience and the international recommendations. Acta Bio Medica: Atenei Parmensis. 2020;91(2):218-21.

3. Corrons JV, De Sanctis V. Rare anaemias, sickle-cell disease and COVID19. Acta Bio Medica: Atenei Parmensis. 2020;91(2):216-7.

4. Sayad B, Rahimi Z. Blood coagulation parameters in patients with severe COVID-19 from Kermanshah Province, Islamic Republic of Iran. East Mediterr Health J. 2020;26(9):999-1004. doi:10.26719/emhj.20.105. [PubMed: 33047789].

5. Nasiri A, Rahimi Z, Vaisi-Raygani A. Hemoglobinopathies in Iran: An Updated Review. Int J Hematol Oncol Stem Cell Res. 2020;14(2):140. [PubMed: 32461799]. [PubMed Central: PMC7231794].

6. Nemati H, Rahimi Z, Bahrami G. The Xmn1 polymorphic site 5' to the (G)gamma gene and its correlation to the (G)gamma:(A)gamma ratio, age at first blood transfusion and clinical features in betathalassemia patients from Western Iran. Mol Biol Rep. 2010;37(1):15964. doi: 10.1007/s11033-009-9566-7. [PubMed: 19444645].

7. Rahimi Z, Ghaderi M, Nagel RL, Muniz A. Prevalence of thrombotic risk factors among beta-thalassemia patients from Western Iran. $J$ Thromb Thrombolysis. 2008;26(3):229-33. doi: 10.1007/s11239-007-01630. [PubMed: 17982733].

8. Chowdhury SF, Anwar S. Management of Hemoglobin Disorders During the COVID-19 Pandemic. Front Med (Lausanne). 2020;7:306. doi: 10.3389/fmed.2020.00306. [PubMed: 32582745]. [PubMed Central: PMC7295899].

9. Bazi A, Shahramian I, Yaghoobi H, Naderi M, Azizi H. The Role of Immune System in Thalassemia Major: A Narrative Review. J Pediatr Rev. 2017;6(2):29-36. doi: 10.5812/jpr.14508.

10. Sasi S, Yassin MA, Nair AP, Al Maslamani MS. A Case of COVID-19 in a Patient with Asymptomatic Hemoglobin D Thalassemia and Glucose-6Phosphate Dehydrogenase Deficiency. Am J Case Rep. 2020;21. e925788. doi: 10.12659/AJCR.925788. [PubMed: 32697769]. [PubMed Central: PMC7394553].

11. Lansiaux E, Pebay PP, Picard JL, Son-Forget J. COVID-19: betathalassemia subjects immunised? Med Hypotheses. 2020;142:109827. doi: 10.1016/j.mehy.2020.109827. [PubMed: 32447232]. [PubMed Central: PMC7217120].

12. Thomas T, Stefanoni D, Dzieciatkowska M, Issaian A, Nemkov T, Hill RC, et al. Evidence of Structural Protein Damage and Membrane Lipid Remodeling in Red Blood Cells from COVID-19 Patients.J Proteome Res. 2020;19(11):4455-69. doi: 10.1021/acs.jproteome.0c00606. [PubMed: 33103907]. [PubMed Central: PMC7640979].

13. Sayad B, Afshar ZM, Mansouri F, Rahimi Z. Leukocytosis and alteration of hemoglobin level in patients with severe COVID-19: Association of leukocytosis with mortality. Health Sci Rep. 2020;3(4):194. doi: 10.1002/hsr2.194. [PubMed: 33083572]. [PubMed Central: PMC7556692].

14. Sayad B, Karimi M, Rahimi Z. Sickle cell disease and COVID-19: Sus ceptibility and severity. Pediatr Blood Cancer. 2021;68(8). e29075. doi: 10.1002/pbc.29075. [PubMed: 34061431]. [PubMed Central: PMC8209850].

15. Karimi M, Haghpanah S, Zarei T, Azarkeivan A, Shirkavand A, Matin S, et al. Prevalence and severity of Coronavirus disease 2019 (COVID19) in Transfusion Dependent and Non-Transfusion Dependent $\beta$ thalassemia patients and effects of associated comorbidities: an Iranian nationwide study. Acta Bio Medica: Atenei Parmensis. 2020;91(3). e2020007.

16. Rahimi Z, Merat A, Gerard N, Krishnamoorthy R, Nagel RL. Implications of the genetic epidemiology of globin haplotypes linked to the sickle cell gene in southern Iran. Hum Biol. 2006;78(6):719-31. doi: 10.1353/hub.2007.0016. [PubMed:17564250]. 
17. Drouin E. Beta-thalassemia may protect against COVID 19. Med Hypotheses. 2020;143:110014. doi: 10.1016/j.mehy.2020.110014. [PubMed: 32615501]. [PubMed Central: PMC7321655].

18. Motta I, Migone De Amicis M, Pinto VM, Balocco M, Longo F, Bonetti F, et al. SARS-CoV-2 infection in beta thalassemia: Preliminary data from the Italian experience. Am J Hematol. 2020;95(8):E1989. doi: 10.1002/ajh.25840. [PubMed: 32311145]. [PubMed Central: PMC7264660].

19. Karimi M, Haghpanah S, Azarkeivan A, Zahedi Z, Zarei T, Akhavan Tavakoli $\mathrm{M}$, et al. Prevalence and mortality in beta-thalassaemias due to outbreak of novel coronavirus disease (COVID-19): the nationwide Iranian experience. Br J Haematol. 2020;190(3):e13740. doi: 10.1111/bjh.16911. [PubMed: 32484906]. [PubMed Central: PMC7300954].

20. de Sanctis V, Canatan D, Corrons JLV, Karimi M, Daar S, Kattamis C, et al. Preliminary Data on COVID-19 in Patients with Hemoglobinopathies: A Multicentre ICET-A Study. Mediterr J Hematol Infect Dis. 2020;12(1). e2020046. doi: 10.4084/MJHID.2020.046. [PubMed: 32670524]. [PubMed Central: PMC7340245].

21. Karimi M, Haghpanah S, Azarkeivan A, Matin S, Safaei A, De Sanctis V. Coronavirus Disease 2019 (COVID-19) Severity in Patients with Thalassemias: A Nationwide Iranian Experience. Mediterr J Hematol Infect Dis. 2021;13(1). e2021008. doi: 10.4084/MJHID.2021.008. [PubMed: 33489047]. [PubMed Central: PMC7813286].

22. Telfer P, De la Fuente J, Sohal M, Brown R, Eleftheriou P, Roy N, et al. Real-time national survey of COVID-19 in hemoglobinopathy and rare inherited anemia patients. Haematologica. 2020;105(11):2651-4. doi: 10.3324/haematol.2020.259440. [PubMed: 33054122]. [PubMed Central: PMC7604629].

23. Dehshal MH, Hosoya S, Bahremani FH, Namini MT, Eleftheriou A. COVID-19 and Thalassaemia in Iran. Thal Rep. 2020;10(1). doi: 10.4081/thal.2020.9157.

24. Marhaeni W, Wijaya AB; Khairiyadi; Munawaroh; Hendriyono. Coagulation Abnormalities Due to COVID-19 in a Child with Thalassemia. Indian J Pediatr. 2021;88(4):1-2. doi: 10.1007/s12098-02003600-9. [PubMed: 33415546]. [PubMed Central: PMC7790717].

25. Okar L, Ali M, Parengal J, Yassin MA. COVID-19 and thalassemia beta major in splenectomized patient: Clinical case progression and literature review. Clin Case Rep. 2020;8(12):1-5. doi: 10.1002/ccr3.3345. [PubMed: 33363851]. [PubMed Central: PMC7752600].

26. Torti L, Maffei L, Sorrentino F, De Fabritiis P, Miceli R, Abruzzese E. Impact of SARS CoV-2 in Hemoglobinopathies with Immune Disfunction and Epidemiology. A Protective Mechanism from Beta Chain Hemoglobin Defects? Mediterr J Hematol Infect Dis. 2020;12(1). e2020052. doi: 10.4084/MJHID.2020.052. [PubMed: 32670530]. [PubMed Central: PMC7340215].

27. Marziali M, Ribersani M, Losardo AA, Taglietti F, Pugliese P, Micozzi A, et al. COVID-19 pneumonia and pulmonary microembolism in a patient with B-thalassemia major. Clin Case Rep. 2020:14. doi: 10.1002/ccr3.3275. [PubMed: 33173583]. [PubMed Central: PMC7646639].
28. Pinto VM, Derchi GE, Bacigalupo L, Pontali E, Forni GL. COVID19 in a Patient with beta-Thalassemia Major and Severe Pulmonary Arterial Hypertension. Hemoglobin. 2020;44(3):218-20. doi: 10.1080/03630269.2020.1779082. [PubMed: 32552139].

29. Wenzhong L, Hualan L. COVID-19: Attacks the 1-Beta Chain of Hemoglobin and Captures the Porphyrin to Inhibit Human Heme Metabolism. ChemRxiv: Cambridge Open Engage. 2020;10(4).

30. Ehsani S. COVID-19 and iron dysregulation: distant sequence similarity between hepcidin and the novel coronavirus spike glycoprotein. Biol Direct. 2020;15(1):1-13. doi: 10.1186/s13062-020-00275-2. [PubMed: 33066821]. [PubMed Central: PMC7563913].

31. Cavezzi A, Troiani E, Corrao S. COVID-19: hemoglobin, iron, and hypoxia beyond inflammation. A narrative review. Clin Pract. 2020;10(2):1271. doi: 10.4081/cp.2020.1271. [PubMed: 32509258]. [PubMed Central: PMC7267810].

32. Read R. Flawed methods in "COVID-19: Attacks the 1-beta chain of hemoglobin and captures the porphyrin to inhibit human heme metabolism. ChemRxiv. 2020. doi: 10.26434/chemrxiv.12120912.v2.

33. Park KC, Donovan K, McKechnie S, Ramamurthy N, Klenerman P, Swietach $\mathrm{P}$. Single-cell oxygen saturation imaging shows that gas exchange by red blood cells is not impaired in COVID-19 patients. $\mathrm{Br} J$ Haematol. 2020;190(4). doi: 10.1111/bjh.17025.

34. Sotoudeh E, Sotoudeh H. A hypothesis about the role of fetal hemoglobin in COVID-19. Med Hypotheses. 2020;144:109994. doi: 10.1016/j.mehy.2020.109994. [PubMed: 32570166]. [PubMed Central: PMC7834262].

35. Brunetta DM, De Santis GC, Silva-Pinto AC, Oliveira de Oliveira LC, Covas DT. Hydroxyurea increases plasma concentrations of microparticles and reduces coagulation activation and fibrinolysis in patients with sickle cell anemia. Acta Haematol. 2015;133(3):287-94. doi: 10.1159/000362148. [PubMed: 25472687].

36. Boelaert JR, Sperber K, Piette J. Chloroquine exerts an additive in vitro anti-HIV type 1 effect when associated with didanosine and hydroxyurea. AIDS Res Hum Retroviruses. 1999;15(14):1241-7. doi: 10.1089/088922299310133. [PubMed: 10505672].

37. Yuksel S, Gulec MA, Gultekin Z, Caglar A, Beytemur O, Alagoz E, et al. Histopathological, immunohistochemical, and biomechanical effects of splenectomy on Achilles tendon healing in rats. Connect Tissue Res. 2019;60(2):200-8. doi: 10.1080/03008207.2018.1483361. [PubMed: 29860899].

38. Yadav U, Pal R. Challenging Times for Children With Transfusiondependent Thalassemia Amid the COVID-19 Pandemic. Indian Pediatr. 2020;57(5):478. [PubMed: 32255436]. [PubMed Central: PMC7240232].

39. Lee CK, Leung JNS, Cheng P, Lung DC, To KKW, Tsang DNC. Absence of SARS-CoV-2 viraemia in a blood donor with COVID-19 post-donation. Transfus Med.2021;31(3):1-2. doi: 10.1111/tme.12724. [PubMed:33015920]. [PubMed Central: PMC7677860].

40. d'Arqom A, G. Putri M, Savitri Y, Rahul Alfaidin AM. Vitamin and mineral supplementation for beta-thalassemia during COVID-19 pandemic. Future Sci OA. 2020;6(9):FSO628. doi: 10.2144/fsoa-2020-0110. [PubMed: 33230422]. [PubMed Central: PMC7434224]. 